\title{
A Bandwidth-Efficient Application Level Framing Protocol for H.264 Video Multicast over Wireless LANs
}

\author{
Abdelhamid Nafaa, Yassine Hadjadj Aoul, Daniel Negru, and Ahmed Mehaoua \\ University of Versailles, CNRS-PRiSM Lab. \\ 45, av. des Etats Unis 78035, Versailles, France \\ Tel: +3313925 40 59, Fax: +33139254057 \\ \{anaf, yana, dan, mea\}@prism.uvsq.fr
}

\begin{abstract}
Optimizing wireless bandwidth utilization is one of the numerous challenges in wireless IP multimedia systems design. This paper describes and evaluates the performance of a novel Application Level Framing protocol for efficient transmission of H.264 video over error-prone wireless IP links. The proposed ALF protocol introduces an innovative loss spreading scheme for video streaming services which is based on $(i)$ a bandwidth-efficient adaptive H.264 video fragmentation and (ii) an unequal-interleaved protection for improving FEC efficiency. Both video fragmentation and interleaving are coordinated in a frame-based granularity providing bounded end-to-end delays. Performance evaluation results show that the proposed protocol allows graceful video quality degradation over error-prone wireless links while minimizing the overall bandwidth consumption and the end-to-end latency.
\end{abstract}

\section{Introduction}

Wireless communication technology has gained widespread acceptance in recent years. The IEEE 802.11b 1 standard has led wireless local area networks (LANs) into greater use, providing up to $11 \mathrm{Mbps}$ of shared bandwidth. With such high bandwidth, the demand for supporting time-sensitive traffic applications, such as video-on demand and interactive multimedia, in wireless LANs has been increasing. Meanwhile, the recently adopted ITU-T H.264 standard 2 (known also as ISO/IEC International Standard 14496 Part 10) achieves efficient video encoding and bandwidth savings. H.264 experts have taken into account transmission over packet based networks in the video codec design from the very beginning. The overall performance of H.264 is as such that bit rate savings of $50 \%$ or more, compared to the current state of technology, are reported. Digital Satellite TV quality, for example, was reported to be achievable at $1.5 \mathrm{Mbit} / \mathrm{s}$, compared to the current operation point of MPEG-2 video at around 3.5 Mbit/s. In this paper, we investigate H.264 video multicast communications over IEEE $802.11 \mathrm{~b}$ wireless LAN. Though the proposed protocol is network independent and can support various media types as well.

In previous work 3, 4, we have addressed wireless video communication issue from an application point of view. Thus, we proposed a multimedia elementary streams classification and aggregation that provides wireless bandwidth savings and packet loss tolerance. However, the intrinsic wireless link characteristics involve unpredictable burst errors that are usually uncorrelated with the instantaneous available bandwidth. The resulting packet losses and bit errors can have devastating effects on multimedia quality. To overcome residual BER (Bit Error Rate), error control mechanisms of video streams is generally required. Error control mechanisms are 
popular on dealing with packet loss and delay over bandwidth limited fading wireless channels. Such mechanisms involve Forward Error Correction (FEC), Automatic Retransmission ReQuest (ARQ), and error resilience tools. FEC has been commonly suggested for real-time applications due to $(i)$ its proven scalability for multicast communications and (ii) the strict delay requirements of media streams.

Typical communications over wireless networks involve high bit error rates that translate to correlated adjacent packets losses. In this case, the classical adaptive FEC approaches 5, 6, can be inefficient since they involve an excessive bandwidth usage. Actually, such approaches use FEC to protect consecutive original data packets, which reduce its effectiveness against burst packets losses. This often implies transmitting additional FEC packets to overcome the increasing BER. Hence, we propose a novel low-delay interleaved FEC protection scheme. The idea is to spread the burst loss before FEC recovering, so that the burst loss manifests itself as a number of disjoint packet losses in the FEC-recovered data stream. This process first consists of adaptively fragmenting the H.264 Frames in order to achieve a better link utilization. The second phase is based on the application of an unequal-interleaved media packet protection, which takes into account the H.264 video Frames relevance. Thus, our protocol minimizes burst errors consequences, as well as the video distortion at receivers' side, while minimizing the overall bandwidth consumption.

The remainder of this paper is as follows. Section 2 investigates reliable H.264 video multicasting over Wireless LAN. Then, the proposed protocol is presented in Section 3. Section 4 is devoted to the performance evaluation. Finally, Section 5 concludes the paper.

\section{Related Works on H.264 Streaming over Wireless LAN}

\subsection{H.264 over Wireless IP Networks}

A new feature of $\mathrm{H} .264$ design 7 resides in the introduction of a conceptual separation between Video Coding Layer (VCL), which provides the core high-compression representation of the video picture content, and Network Adaptation Layer (NAL), which packages that representation for efficient delivery over a particular type of network.

The H.264 NAL design provides the ability to customize the format of the VCL data for delivery over a variety of particular networks. Therefore, a unique packetbased interface between the VCL and the NAL is defined. The packetization and appropriate signaling are part of the NAL specification, which is not necessarily part of the H.264 specification itself. For the transmission of video over WLANs with limited bandwidth and transmission power resources, the necessity for high compression efficiency is an obvious task. Besides, adaptation of the video data to the network fluctuation is an additional important task due to special properties of the wireless channel. These two design goals, compression efficiency and network friendliness, motivate the differentiation between the VCL for coding efficiency and the NAL to take care of network issues. In the H.264 framework, all information that was traditionally conveyed in sequences, group-of-picture, or picture headers is conveyed out of band. During the setup of the logical channel the capability exchange takes place. This procedure was already subject to many discussions within H.264, and it was agreed that a simple version/profile/level concept should be used; current work in the IETF 9 is underway to enable such features. 


\subsection{Reliable Multicast Communications over Wireless LAN}

In order to reliably communicate over packet-erasure channels, it is necessary to exert some form of error control 8. Two classes of communication protocols are used in practice to reliably communicate data over packet networks: synchronous and asynchronous. Asynchronous communication protocols, such as ARQ operates by dividing the data into packets and appending a special error check sequence to each packet for error detection purposes. The receiver decides whether a transmission error occurred by calculating the check sequence. For each intact data packet received in the forward channel, the receiver sends back an acknowledgement. While this model works very well for data communication, it is not suitable for multimedia streams with hard latency constraints. The maximum delay of the ARQ mechanism is unbounded, and in the case of live streaming it is necessary to interpolate late-arriving or missing data rather than insert a delay in the stream playback. In synchronous protocols (i.e. FECbased protocols), the data are transmitted with a bounded delay but generally not in a channel adaptive manner. The FEC codes are designed to protect data against channel losses by introducing parity packets. No feedback channel is required. If the number of lost packets is less than the decoding threshold for the FEC code, the original data can be recovered perfectly.

ARQ-based schemes are not appropriate for the multicast case for three reasons: $(i)$ ACK explosion, that scales with the multicast group size; (ii) for significant loss rate, each user will require frequent packet retransmissions that are probably useless for the other multicast clients; and (iii) unbounded data transmission delays. Hence, using a FEC-based error control seems to be more appropriate for real time multicast communication.

The IEEE 802.11 standard 1 uses the same logical link layer as other 802-series networks (including the 802.3 wired Ethernet standard), and uses compatible 48-bit hardware Ethernet addresses to simplify routing between wired and wireless networks. As in the wired Ethernet, corrupted packets are dropped at the link layer (i.e. the packets with bit errors are unavailable to a multimedia application). The communication is complicated by the inability of radio transceivers to detect collisions as they transmit, and the potential for devices outside the network to interfere with network transmissions. Communication is also hampered by the hidden node problem. Therefore, the IEEE $802.11 \mathrm{~b}$ standard uses a complex MAC protocol to cope with these wireless communication specificities. The basic medium access protocol is a DCF (Distributed Coordination Function) that allows medium sharing through the use of CSMA/CA (Carrier Sense Medium Access / Collision Avoidance). In addition, all directed traffic uses immediate positive acknowledgment (ACK frame) where retransmission is scheduled by the sender if no ACK is received. That is, within IEEE $802.11 \mathrm{~b}$ unicast communications, all wireless data frames are acknowledged by the receiver. Furthermore, each retransmission introduces an additional latency due to triggering of the collision avoidance routine. The sender uses limited retransmission persistence, so the data can be dropped at the source after several retransmission attempts. In case of multicast or broadcast traffic, however, the data packets are not acknowledged, and hence no retransmission is performed on the MAC/Logical link layer; this mode of communication reduces transmissions delays while making communications less reliable. 


\subsection{Specific Related Works}

Nowadays, most of the reliable multicast video distribution protocols propose the use of ARQ (see 10 and references therein). Besides, FEC for multicast streaming of different characteristic have been extensively studied in the literature 6,12 . In a multicast scenario, to tackle the problem of heterogeneity and to ensure graceful quality degradation, the use of multi resolution-based scalable bitstreams has been previously suggested in 10 and 13. These approaches are, however, dedicated to multilayer video coding throughout their design.

In wireless communications, packet loss can exhibit temporal dependency or burstiness. For instance, if packet $n$ is lost, packet $n+1$ is also likely to do so. This translates to burstiness in network losses, which may worsen the perceptual quality compared to random losses at the same average loss rate. As a consequence, the performance of FEC is affected, e.g., percentage of packets that cannot be recovered. Moreover, the final loss pattern after FEC recovering could be even burstier due to the dependency between losses, which affects audio/video quality and effectiveness of loss concealment. In order to reduce burst loss, redundant information has to be added into temporally distant packets, which introduces even higher delay. Hence, the repair capability of FEC is limited by the delay budget 14. Another sender-based loss recovery technique, interleaving, which does not increase the data rate of transmission, also faces the same dilemma. The efficiency of loss recovery depends on over how many packets the source packet is interleaved and spread. Again, the wider the spread, the higher the introduced delay.

In this paper, we introduce a novel technique that combines reliability and efficiency advantages of both interleaving and FEC coding. Our proposal is based on an adaptive H.264 streams fragmentation coordinated with an unequal-interleaved protection scheme. Thus, we improve the error resiliency while minimizing the overall bandwidth consumption and still meeting the delay constraints.

\section{Unequal Interleaved FEC Protocol for Reliable Wireless Video Multicasting}

\subsection{Adaptive H.264 Video Fragmentation and Packetization}

Basically, mobile devices are hand-held and constrained in processing power. In addition, the mobile environment is characterized by harsh transmission conditions in terms of fading and multi-user interference, which results in time- and locationvarying channel conditions. Therefore, a mobile video codec design must minimize terminal complexity while still remaining efficient. Consequently, in our work we specially focus on H.264 Baseline Profile to reduce the receiver's decoder complexity. In this H.264 coder release the data partitioning features are not enabled.

Internally, the NAL uses NALU (NAL Units) 9. A NAL unit consists of a one-byte header and the payload byte string. A complete separation between the VCL and the NAL is difficult to obtain because some dependencies exist. The packetization process is an example: error resilience, in fact, is improved if the VCL is instructed to create slices of about the same size of the packets and the NAL told to put only one slice per packet. The error resilience characteristics of the transmission will take profit of it because all the data contained in a certain packet can be decoded independently from the others. Note that in H.264, the subdivision of a Frame into slices has not to 
be the same for each Frame of the sequence; thus the decoder can flexibly decide how to make the slices. However, they should not be too short because a decrease of the compression ratio would occur for two reasons, i.e. the slice headers would reduce the available bandwidth and the context-based entropy coding would become less efficient. Moreover, in wireless channels, the size of transmitted packets influences its error probability; longer packets are more likely to contain transmission errors 1516 . In this paper the trade-off involved in the packet creation process will be investigated, studying the performances of the video transmission as a function of the packet size. Actually, we try to find, for each Frame to be transmitted, the optimal slice size that maximizes the bandwidth utilization taking into account the fluctuating wireless link conditions (it is assumed that a NALU corresponds to a Slice).

Let FRAMEsize be the Frame size, $\mathrm{S}$ the packet size in bytes, oh the header size in bytes and $L r$ the wireless channel loss rate. The link utilization, $\mathrm{U}$, is then given by (1). U represents the original video data over the transmitted data (i.e. including the FEC redundancy).

$$
U=\frac{\text { FRAMEsize }}{\left(\frac{\text { FRAMEsize }}{S-o h}+\left\lceil\frac{\text { Lr.FRAMEsize }}{S-o h}\right\rceil\right) \cdot S}
$$

Where, $N A L U$ size $=S-o h$ and $o h+1 \leq S \leq M T U$ In Fig. 1, $U$ is plotted against $S$ for $F R A M E S i z e=10000$ and $o h=40$ at four different loss rates (i.e. $L r=\{0,0.05,0.2$ and $0.3\})$.

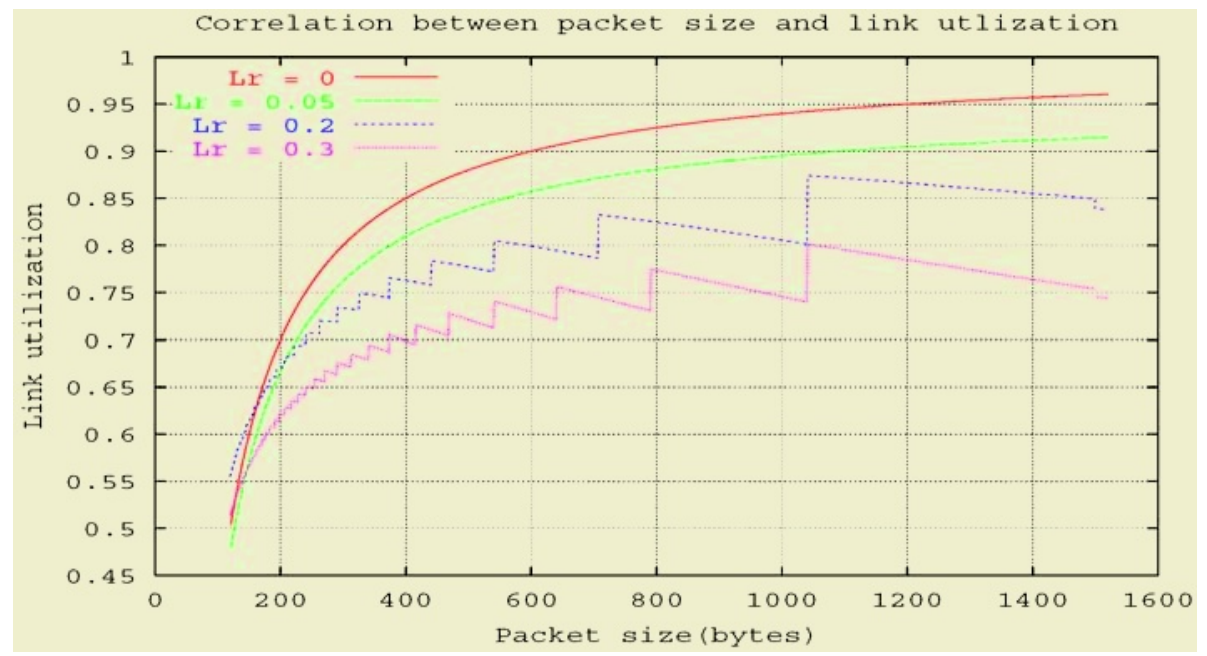

Fig. 1. Correlation between packet size and link utilization.

Fig. 1 depicts the wireless channel utilization for different packet loss rates. It is quite obvious that a small packet size provides better performance when the channel loss rate is too high. In the other hand, systematically choosing a small packet size does not necessarily give good channel utilization. Now, it is readily realized that the maximum of the utilization function is obtained by resolving the equation for $\mathrm{S}$. Thus, NALUSize is determined for each Frame to be transmitted based on the measured loss 
rate. The fragmentation/encapsulation presented here, provides better wireless link utilization. Moreover, this scheme minimizes the dependency between adjacent RTP packets, which mitigate the dependency of H.264 decoder on any lost packet.

\subsection{Unequal Interleaved FEC Protection Protocol}

Fig. 2 illustrates a sample trace we obtained using an 802.11 AP (Access Point) and wireless receivers. Each point represents the packet loss rate measured at each receiver; we emphasize the channel behavior when multicasting video over wireless links. The AP sent multicast H.264 video packets and the receiving stations recorded the sequence number of the correctly received packets. It should be noted that this experience reveals an important number of adjacent packet losses as a consequence of the wireless burst errors. Furthermore, the packet loss rate is different at each receiver due to location-varying channel conditions.

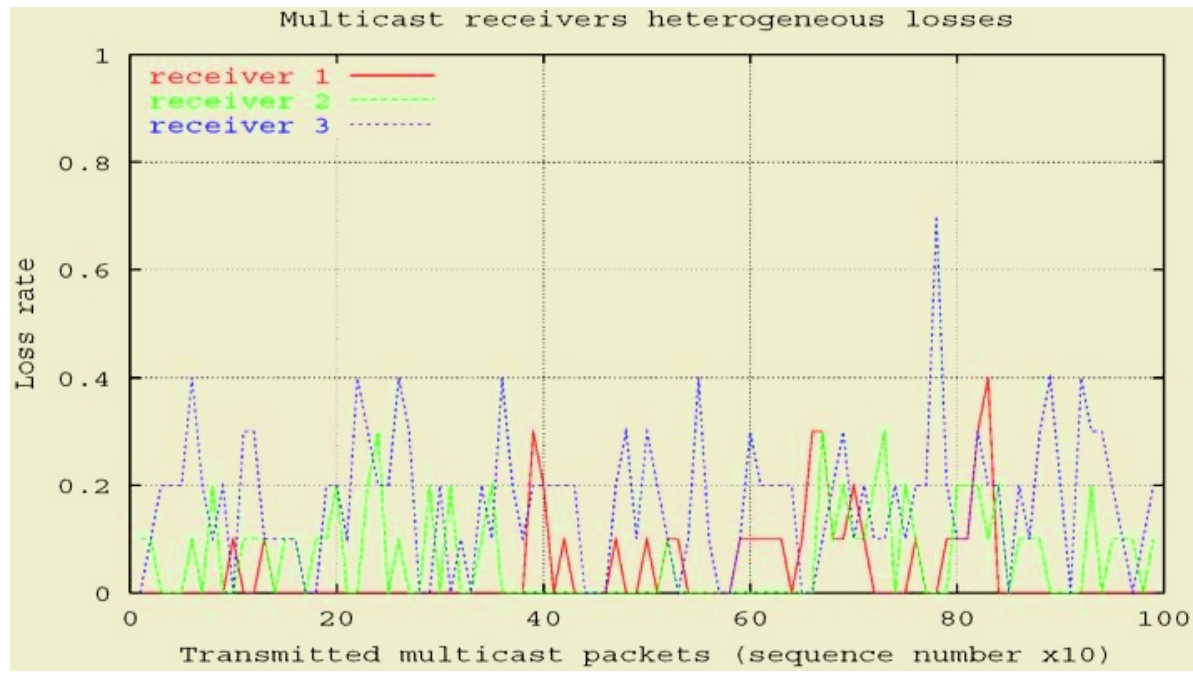

Fig. 2. Correlated multicast receivers packet losses.

Since the wireless link fluctuation occurs usually through unpredictable adjacent packets losses, we propose to use an interleaved FEC protection. As depicted in Fig. 3, the redundant FEC packets protect temporally scattered RTP packets in order to cope with wireless sporadic packet losses. This increases the FEC efficiency through improving the error resiliency at clients' side.

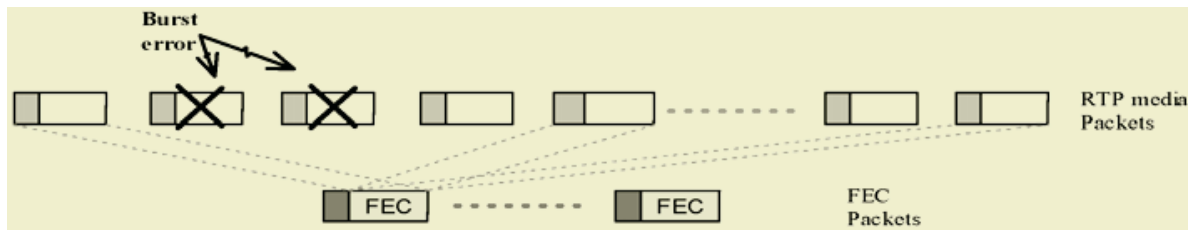

Fig. 3. Scattered video packets protection. 
Within UI-FEC, the time is divided into transmission rounds. A transmission round ends when the sender transmits the last packet of a Frame. Each Frame is divided into several UBs (Unequal loss protection Block). An UB consists of $n=k+h$ packets (see Fig. 5). At this point, we define the interleaving factor $(i)$ as the Sequence Number (SN) difference between each two successive protected RTP packets in the UB. The interleaving factor is fixed for the $k$ protected RTP packets belonging to the same $\mathrm{UB}$ (see Eq.2). When $i=1$, the interleaved protection is not applied and, hence, the FEC packets protect consecutive RTP packets. Fig. 4 summarizes the UI-FEC protocol working example for an interleaving $i=3$. In this case, the adjacent video packets (in terms of transmission order) are protected in different FEC blocks. Here, the interleaving factor represents the interleaving stride ${ }^{1}$.

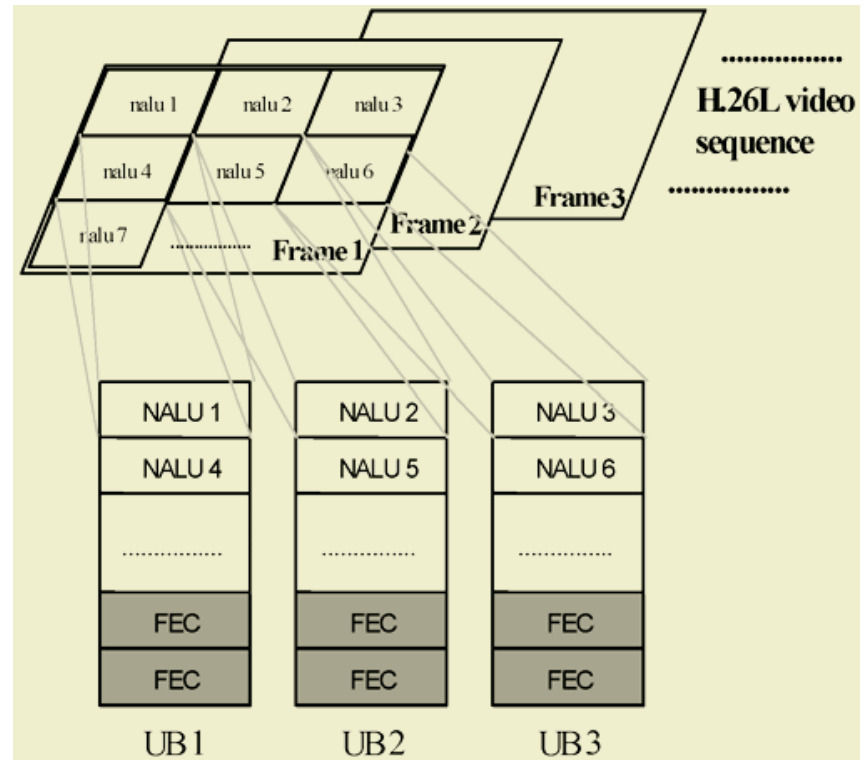

Fig. 4. UI-FEC Protocol.

Note that for a given Frame $(\mathrm{F})$, the interleaving factor $(i)$ represents the number of UBs constituting F. Moreover, $i$ is fixed for all UBs belonging to the same Frame. For synchronization consideration the protected media data of a given UB must belong to the same Frame. In other words, each Frame is transmitted as an integer number of UBs. After applying FEC, the media packets are transmitted in their initial order (i.e. according to the sequence number order). Note that the delays introduced by the FEC interleaving do not have important consequences, since the interleaving is applied over a single Frame. The induced delays can be resolved through an initial buffering time. This novel interleaving schema over a single Frame is used to provide an adaptive and unequal FEC protection.

1 By interleaving stride, we mean the separation (in terms of packet transmission order) between two consecutive data packets in the same FEC block. This is useful in spreading the burst loss, so that the burst loss manifests itself as a number of disjoint packet losses in the FEC-recovered data stream. 


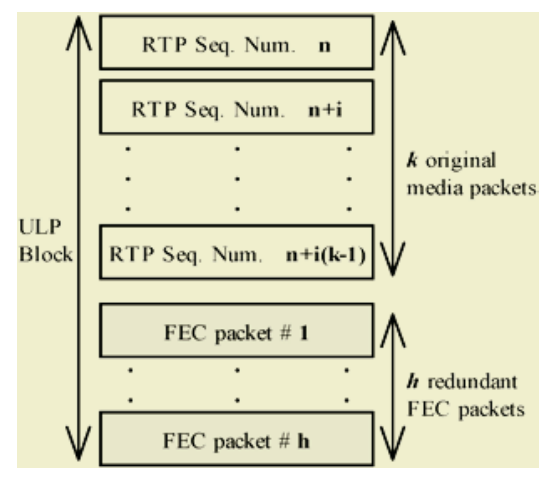

Fig. 5. UB structure details.

For FEC efficiency 6, it is suitable to keep the number of information packets $(k)$ as high as possible with few redundant packets. Actually, a higher number of information packets leads to better granularity, which allows adjusting the redundancy rate more precisely according to wireless channel conditions. As an example, if a Frame to be transmitted has a size of 10 Kbytes, a packet payload size (NALUSize) of 1 Kbyte will result in 10 RTP packets. This makes the precision at which the error protection can be applied in units of $10 \%$.

In our study, we take a minimum of 10 information packets for each UB (i.e. guarantying a minimum precision of $10 \%$ ). This is insured using an appropriate $1 \geq 10$ taking into account the mean Frame size (see formula (2)). For a fixed 1, both interleaving factor $(i)$ and the number $(k)$ of media packet per UB are then easily stated; where the FRAMESize is obtained from the Frame header, while the NALUSize is fixed by our H.264 video packetization process (see section 3.1).

$$
k=\left\lfloor\frac{\text { FRAMESize }}{i * \text { NALUSize }}\right\rfloor, \text { where, } i=\left\lfloor\frac{\text { FRAMESize }}{l * \text { NALUSize }}\right\rfloor
$$

It is clear that the interleaving factor is tightly dependent on Frame size. Since the different Frames (pictures) of a coded H.264 video sequence have a different size, the interleaving factor $(i)$ scales along with the Frame size. Consider that an intra-coded picture (picture-I) is larger than an inter-coded picture (picture-P or picture-B). Basically, the large inter-coded picture conveys a lot of motion vectors and error prediction information. Otherwise, the larger the inter-coded picture is, the more it codes a highly changeful scene with different texture, and the more it involves an important video distortion when lost. As a consequence, within our UI-FEC, the interleaving protection is applied differently based on Frame size and hence Frame relevance, which provides a better loss recovery for the most sensitive H.264 bitstream. Thus, the different Frames are interleaved unequally, protected, then transmitted based on their relevance and without using any previous video stream classification scheme.

The adaptive FEC scheme proposed in this paper is based on systematic $\operatorname{RS}(n, k)$ codes, where $n, k$ and $i$ are reassigned for each Frame to be transmitted based on the relevant priority of the Frame and the current loss rate of the communication channel. When using $k$ source data in an UB, transmitting $h$ FEC packets provides an erasure resiliency against a packet loss rate of $h / n$. Therefore, it is easy to calculate the 
amount of redundant packets $(h)$ using the packet loss rate $(p)$ and the number $(k)$ of original media packets of current UB to be transmitted (see formula (3)).

$$
h=\frac{p \cdot k}{1-p}
$$

The amount of redundancy introduced is computed once per each Frame to be transmitted depending on $(i)$ current transport-level packet loss rate (i.e. measured before FEC recovering) and (ii) video fragmentation parameters. This is achieved according to (1) and (3).

\subsection{UI-FEC Signaling}

In this section, we highlight the key components involved by UI-FEC deployments. Since the successive UBs will have different structure, we propose a simple signaling of required parameters for UB decoding (see Fig. 6). We transmit together within FEC stream (i.e. FEC header) the base RTP sequence number $(B S e q)$ of the protected RTP packets, the UB size $(n)$, the protected original data $(k)$, and the interleaving factor $(i)$. Thus, both interleaving and redundancy are adjusted to match the overall measured BER.

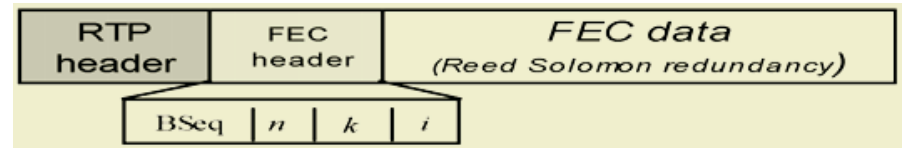

Fig. 6. FEC parameters signaling.

This signaling is sufficiently flexible to provide an adaptive error control based on the signaled FEC interleaving parameters. In addition, the original media stream is decoupled from the FEC stream and its associated parameters, which allows clients without FEC capabilities to decode the original media stream.

\section{Performance Evaluation}

UI-FEC is evaluated with Network Simulator v2 using different network configurations. We emphasize the robustness and efficiency of our proposal with the classical FEC protection (i.e. non interleaved FEC protection) for wireless multimedia communications.

\subsection{Simulation Model}

Video multicasting applications are considered for the simulation. With our proposed technique, the H.264 multicast server generates FEC packets that protect interleaved RTP media packets, while, with classical approach, FEC protection is applied to consecutive RTP media packets. It should be noted that both approaches are evaluated for the same network configurations and using the same amount of FEC protection as well. In our simulation, we use a QCIF Foreman H.264 coded sequence with a constant quantization parameter of 10 (sequence parameters are depicted in Table1); the 
video sequence was generated using the current release of the TML software, JM80. We choose a highly changeful video sequence in order to highlight the unequalinterleaved protection efficiency.

Table 1. Source Video Statistics.

\begin{tabular}{|c|c|c|c|}
\hline $\begin{array}{c}\text { Original } \\
\text { video }\end{array}$ & $\begin{array}{c}\text { Video } \\
\text { configuration }\end{array}$ & $\begin{array}{c}\text { Average } \\
\text { bit-rate }\end{array}$ & $\begin{array}{c}\text { Frame } \\
\text { frequence }\end{array}$ \\
\hline $\begin{array}{c}\text { Foreman } \\
\text { (13.33 seconds) }\end{array}$ & $\begin{array}{c}\text { H. } 264 \\
\text { Baseline Profile }\end{array}$ & $1783 \mathrm{~Kb} / \mathrm{s}$ & $30 \mathrm{Frame} / \mathrm{s}$ \\
\hline
\end{tabular}

We use the network simulation models depicted in Fig. 7 for evaluating and comparing our proposal with the classical approach. The MPEG-4 sender attached to the node " 1 " transmits a multicast MPEG-4 stream to the wireless receivers " 5 " and "7". We include constant-bit-rate (CBR) traffic over UDP to allow loading the network differently each time in order to get further information about UI-FEC behavior.

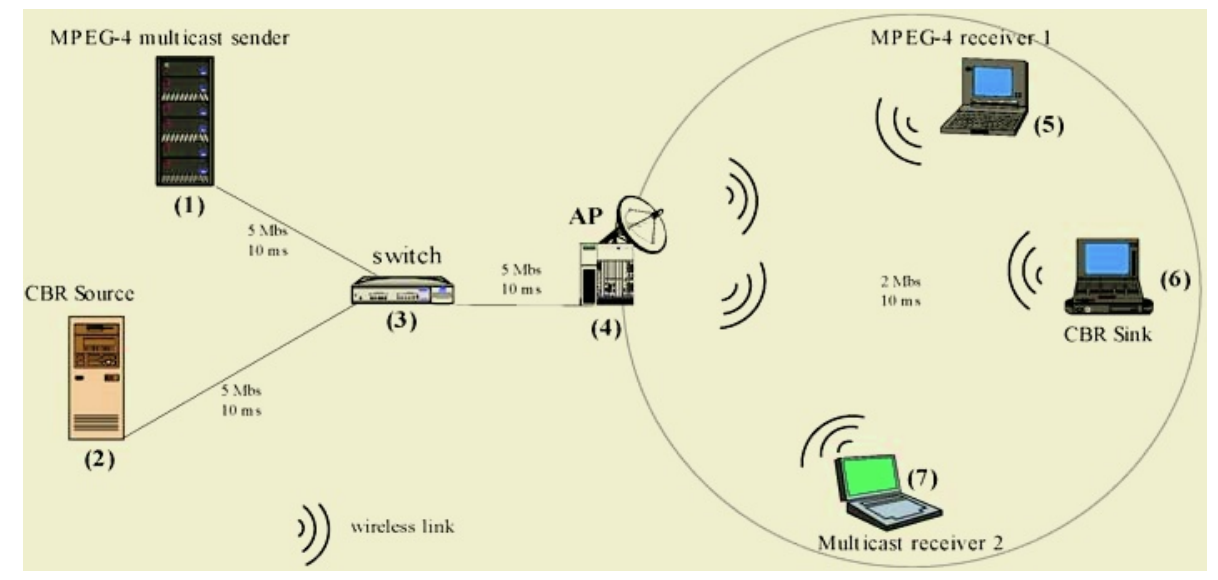

Fig. 7. Network model.

\subsection{Results Analysis}

Fig. 8 represents the final packet loss rates measured for each received H.264 Frame after recovering with FEC; it reveals relatively high bit error rates due to (1) wireless channel burst errors and (2) absence of MAC-level retransmissions in multicast communications. Consider that the measured high loss rates are often provoked by temporally consecutive packet losses affecting the same Frame. We observe that for the same network conditions, UI-FEC increases error resiliency at receivers' side through recovering more RTP packets.

Fig. 9 illustrates the bandwidth consumption measured during the simulation (i.e. for 400 Frames). UI-FEC is more bandwidth efficient than the classical FEC; the measured mean bandwidth saving is around $76 \mathrm{Kbps}$. This bandwidth saving is principally due to a better error resiliency, which implies a reduced FEC transmission. We observed that the unequal-interleaved FEC scheme provides better robustness in net- 
works with a high BER and, consequently, a likely small MTU size. Moreover, UIFEC behaves better when transporting high bit-rate video streams (i.e. video streams with a large mean Frame size).

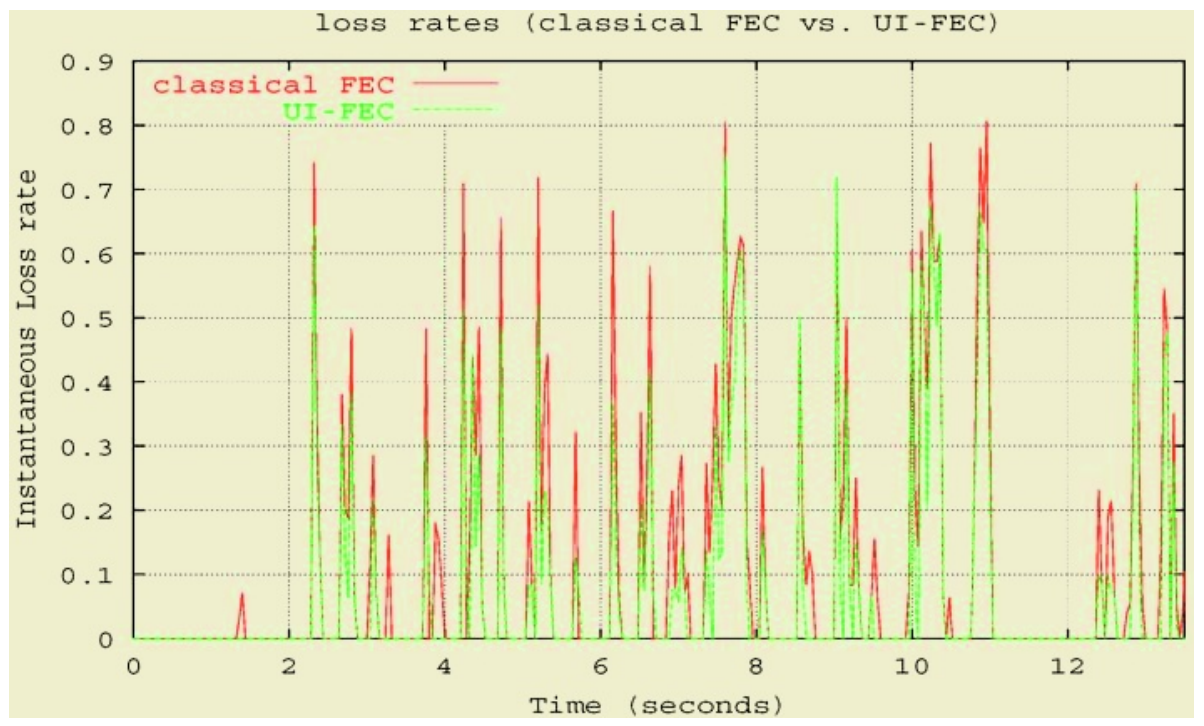

Fig. 8. Instantaneous loss rates.

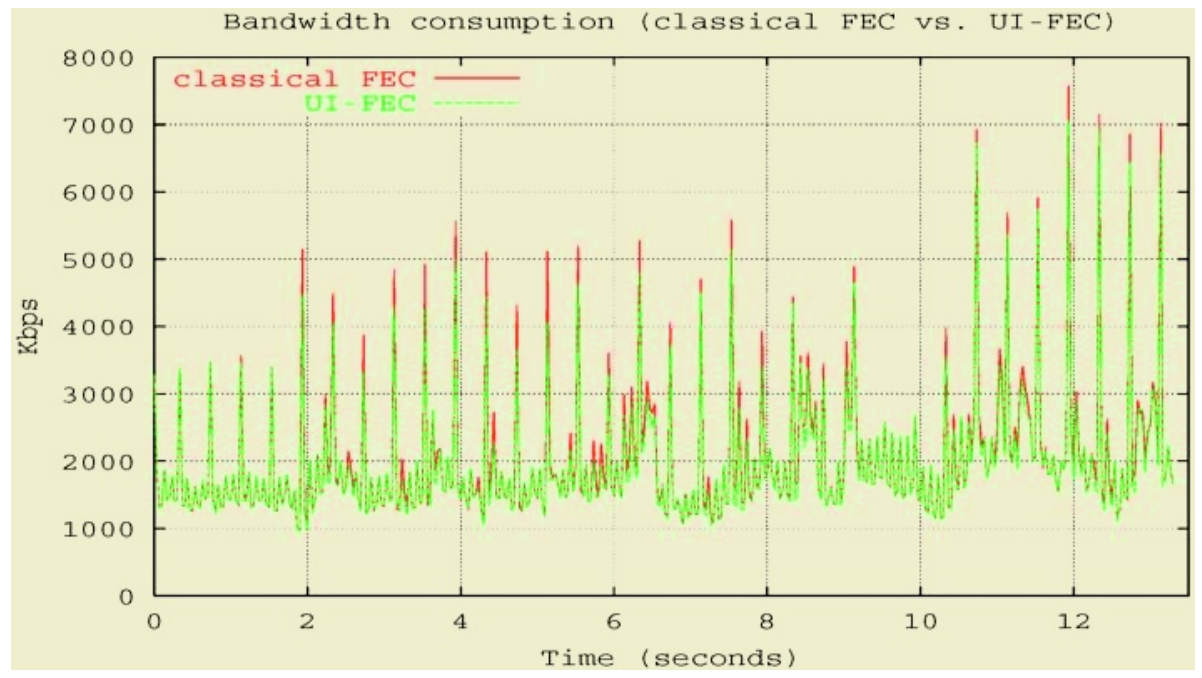

Fig. 9. Instantaneous bandwidth consumption.

We experienced 4 dropped Frames that can not be decoded when transmitting video protected by the classical adaptive FEC, whereas we were able to decode the whole video sequence (400 Frames) through using UI-FEC protocol. Fig. 10 depicts the objective quality measurements of reconstructed video streams when transmitted 
and protected using both FEC techniques. It is clear that UI-FEC protection achieves smoother video quality degradation than a classical FEC protection; the average PSNR gain over the whole communication is around $2.18 \mathrm{~dB}$.

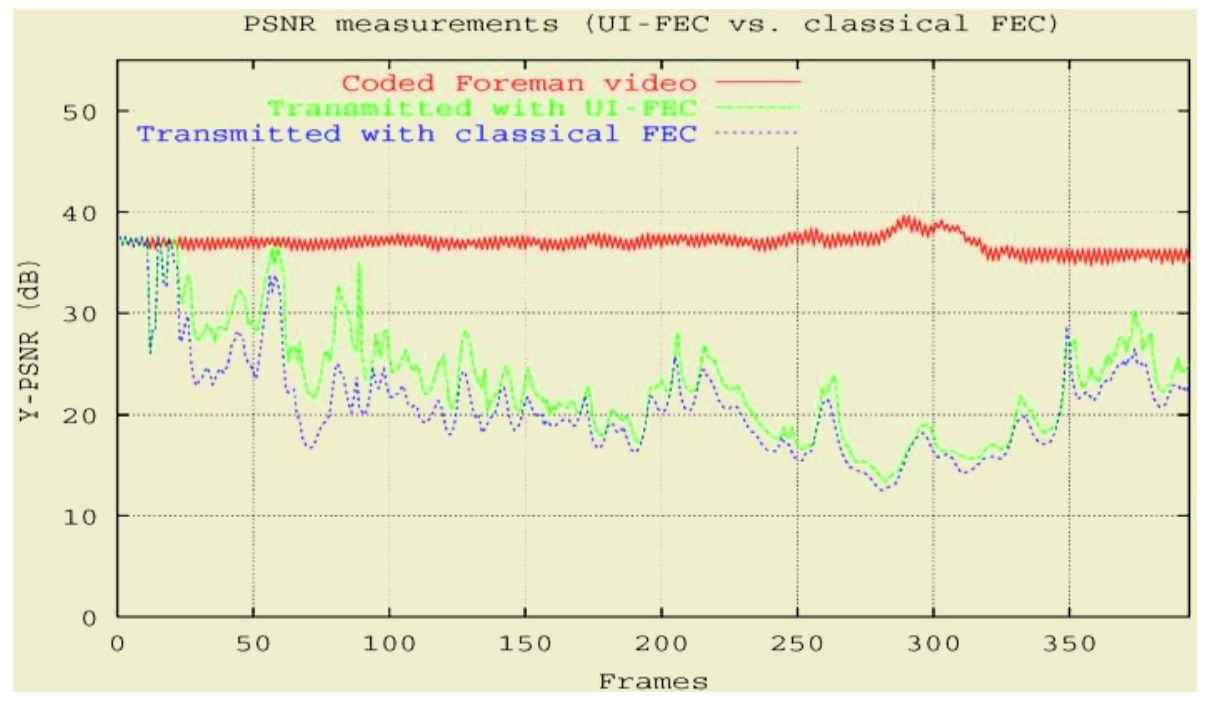

Fig. 10. Objective video quality measurements.

\section{Conclusion}

In this paper we designed, implemented and tested a new Application Framing Protocol named UI-FEC to cope with the problem of efficiently stream real-time compressed video over error-prone wireless links. A distinct feature of UI-FEC is the efficient wireless bandwidth management by means of an adaptive video stream fragmentation coupled with an unequal-interleaved FEC protection. UI-FEC performance was evaluated by simulation with an H.264 multicast distribution service. Results analysis show that UI-FEC offers considerable gains over conventional adaptive FEC in effectively protecting sensitive H.264 video frames, and consequently improving both bandwidth utilization and end-user perceived video quality.

\section{References}

1. Information technology, Wireless LAN medium access control (MAC) and physical layer (PHY) specifications, IEEE 802.11 standard, (August 1999).

2. "Draft ITU-T Recommendation and Final Draft International Standard of Joint Video Specification", (ITU-T Rec. H.264 | ISO/IEC 14496-10 AVC), (May 2003).

3. A. Nafaa, T. Ahmed, Y. Hadjadj-Aoul, A. Mehaoua., RTP4mux: a novel MPEG-4 RTP payload for multicast video communications over wireless IP, in Proc. of IEEE/PV'03,13 ${ }^{\text {th }}$ International Packet Video Workshop, Nantes, France, (April 2003). 
4. T. Ahmed, A. Nafaa, A. Mehaoua, An object-based MPEG-4 multimedia content classification model for IP QoS differentiation, in Proc. of IEEE ISCC'03, IEEE International Symposium on Computer Communications 2003, Turkey, (July 2003), 1091 - 10962

5. T. Ahmed, V. Lecuire, A. Mehaoua, MPEG-4 AVO streaming over IP using TCP-Friendly and unequal error protection, in Proc. of IEEE ICME'03, IEEE Intentional Conference on Multimedia and Expo 2003, Baltimore, USA, (July 2003), 317 - 320.

6. N. Nikaein, H. Labiod, C. Bonnet, MA-FEC: A QoS-based adaptive FEC for multicast communication in wireless networks, in Proc. IEEE ICC'00, IEEE International Conference on Communications 2000, New Orleans, USA, (Jun 2000), 954 - 958.

7. R. Schäfer, T. Wiegand, H. Schwarz, The emerging H.264/AVC standard, EBU Technical Review, (January 2003).

8. S. Lin and D.-J. Costello, Error Control Coding: Fundamentals and Applications, PrenticeHall, Inc. Englewood Cliffs, N.J., (1983).

9. S. Wenger et M.M. Hannuksela, T. Stockhammer, M. Westerlund, D. Singer, RTP payload format for H.264 video, IETF Draft, version 10, expires (January 2005).

10. A. Mjumdar et al., Multicast and unicast real-time video streaming over wireless LANs, IEEE Transaction on Circuits and Systems for Video Technology, Issue: 6, 524 - 534, (June 2002).

11. Nafaa, A. Mehoua "A novel unequal interleaved protection for robust H.264 video multicasting” Technical report, http://prism.uvsq.fr/ anaf/tr02.pdf, (July 2003).

12. J. Nonnenmacher, E. Biersack, and D. Towsley, Parity-based loss recovery for reliable multicast transmission, IEEE/ACM Transactions on Networking, (August 1998), 349-361.

13. G. Liebl, M. Wagner, J. Pandel, and W. Weng, An RTP payload format for erasure-resilient transmission of progressive multimedia streams, IETF Draft, draft-ietf-avt-uxp-05.txt, expires (Sept. 2003).

14. J.-C. Bolot, End-to-end packet delay and loss behavior in the internet, ACM SIGCOMM '93, San Francisco, CA, USA, (September 1993).

15. M. Johanson, Adaptive Forward Error Correction for real-time Internet video, in Proc. of IEEE/PV'03, 13th International Packet Video Workshop, Nantes, France, (April 2003).

16. P. Lettieri and M. B. Srivastava, Adaptive frame length control for wireless link throughput, range, and energy efficiency, in Proc. of IEEE INFOCOM'98, Conference on Computer Communications, San Francisco, CA, (April 1998), 564 - 571. 